\title{
Zeb1 for RCP-induced oral cancer cell invasion and its suppression by resveratrol
}

\author{
Jin Young Kim', Kyung Hwa Cho', Bo Young Jeong', Chang Gyo Park ${ }^{1}$ and Hoi Young Lee
}

\begin{abstract}
Rab coupling protein (RCP) is upregulated in head and neck squamous cell carcinoma (HNSCC) and is correlated with the progression and survival of patients. However, the role of RCP in one of the aggressive types of HNSCC, oral squamous cell carcinoma (OSCC), remains elusive. In the present study, we identified the important role of Zeb1 in RCP-induced OSCC epithelial-to-mesenchymal transition (EMT) and invasion. RCP induces Zeb1 expression, and silencing Zeb1 expression significantly inhibits RCP-induced OSCC invasion. In addition, Zeb1 upregulates MT1-MMP expression to promote OSCC EMT and invasion. Furthermore, we observed that the $\beta 1$ integrin/EGFR/ $\beta$-catenin signaling cascade mediates RCP-induced Zeb1 expression to promote OSCC invasion. Notably, we provide evidence that resveratrol (REV) strongly inhibits RCP-induced Zeb1 expression through blocking $\beta 1$ integrin endosome recycling and EGFR activation, leading to suppression of RCP-induced OSCC invasion, demonstrating the important role of RCP in OSCC invasion and its reversion by REV. Collectively, the present study provides evidence for the first time that RCP aggravates OSCC invasion through increasing Zeb1 expression and subsequently upregulating MT1-MMP expression and that this process is reversed by REV, providing novel biomarkers and indicating the therapeutic potential of REV in OSCC.
\end{abstract}

\section{Introduction}

Head and neck squamous cell carcinoma (HNSCC) stems from the epithelia of the aerodigestive tract, including the oral cavity ${ }^{1}$. The prevalence of HNSCC is increasing worldwide, with nearly 800,000 new diagnoses and 350,000 cancer-associated deaths annually ${ }^{1-3}$. Similar to other types of cancer, metastasis to distant organs is the most common cause of death in HNSCC patients. Metastasis is a complex multistep process that includes epithelial-mesenchymal transition (EMT), which is highlighted by reduced expression of E-cadherin through upregulation of EMT-activating transcription factors (EMT-TFs), such as Zeb proteins ${ }^{4,5}$.

Identified as a Rab4 and Rab11 effector protein, Rab coupling protein (RCP) originates from the RAB11FIP1 gene and is associated with the proliferation and progression of various cancers ${ }^{6,7}$. In particular, RCP is

\footnotetext{
Correspondence: Hoi Young Lee (hoi@konyang.ac.kr)

'Department of Pharmacology, College of Medicine, Konyang University,

Daejeon, Republic of Korea
}

overexpressed in numerous types of cancer, including HNSCC, and augments cancer invasion and metastasis ${ }^{8-11}$. Mechanistically, RCP promotes cancer cell EMT and metastasis by protecting $\beta 1$ integrin from lysosomal digestion and activating $\mathrm{EGFR}^{6,11}$. In addition, RCP participates in EphA2 trafficking during cell repulsion ${ }^{12}$ and mediates mutant $\mathrm{p} 53$-induced cancer invasiveness ${ }^{13,14}$. However, RCP has also been suggested as an important negative regulator in Her-2-positive breast cancer through the lysosomal degradation of Her- $2^{15}$.

In the present study, we investigated the effect of RCP on one of the aggressive types of HNSCC, oral squamous cell carcinoma $(\mathrm{OSCC})^{16}$, and identified that RCP induces OSCC EMT and invasion through Zeb1 and MT1-MMP expression. More interestingly, we revealed that the natural phytoalexin resveratrol (REV) efficiently attenuates RCP-induced OSCC invasion through the downregulation of $\beta 1$ integrin and Zeb1 expression, providing novel biomarkers and potential therapeutic options in OSCC. 


\section{Materials and methods}

\section{Cell culture and reagents}

OSCC cell lines YD-9 and YD-38 were obtained from the Korean Cell Line Bank (Seoul, Korea). YD-10B cells were a gift from Dr. J.I. Yook (Yonsei University College of Dentistry, Seoul, Korea). Oral cancer-associated fibroblasts (CAFs) were generously provided by Dr. X.L. Zhang (Yonsei University College of Dentistry, Seoul, Korea). Oral CAFs were cultured in DMEM and F-12 Ham mixed in a 3:1 ratio and supplemented with $10 \%$ FBS and 1\% penicillin/streptomycin. All OSCC cells were cultured in RPMI supplemented with $10 \%$ FBS and $1 \%$ penicillin/ streptomycin at $37^{\circ} \mathrm{C}$ under $5 \% \mathrm{CO}_{2}$ in a humidified incubator. Gefitinib was purchased from Selleckchem (Houston, TX). REV was purchased from Sigma-Aldrich (St Louis, MO). All other reagents were of the purest grade available.

\section{siRNA and plasmid transfection}

Cells were transiently transfected using Lipofectamine 3000 or RNAiMAX (Invitrogen, Carlsbad, CA) as described previously ${ }^{11}$. RCP and Zeb1 constructs were in the pEGFP-C3 and pCMV6A vectors, respectively. $\beta 1$ integrin $\mathrm{cDNA}$ was added to the pcDNA3 vector. Each empty vector was used as the negative control. RCP and $\beta 1$ integrin constructs were provided by Jim Norman (Beaston Cancer Institute, Glasgow, UK) and Dr. Y.S. Lee (Ewha Woman's University, Seoul, Korea), respectively. The vector for Zeb1 was purchased from Switchgear Genomics (Carlsbad, CA). siRNAs against RCP, Zeb1, $\mathrm{N}$-cadherin, and $\beta 1$ integrin were purchased from SigmaAldrich, and $\beta$-catenin siRNA was purchased from Santa Cruz Biotechnology (Dallas, Texas). Control scrambled siRNA was purchased from Invitrogen. Lentivirus packaging and transduction were performed with a Second Generation packaging mix kit (Abm, Richmond, BC, Canada) according to the manufacturer's protocol. Briefly, the recombinant lentivirus was produced by transfecting HEK293T cells with expression and packaging vectors. The supernatant medium was harvested twice at $24 \mathrm{~h}$ and $48 \mathrm{~h}$. The prepared YD-10B cells were transduced with the virus-containing supernatant with polybrene $(5 \mathrm{mg}$ / $\mathrm{ml}$, Merck Millipore, Darmstadt, Germany). The permanent RCP transfectants were selected using puromycin $(1 \mu \mathrm{g} / \mathrm{ml}$, Thermo Fisher Scientific Inc., Rockford, IL) for 7 days, and the expression of RCP was evaluated by immunoblotting.

\section{Quantitative RT-PCR}

Isolation of total cellular RNA and subsequent analysis were performed as previously described ${ }^{11,17}$. Complementary DNA was amplified using an iQ5 RealTime PCR Detection System (Bio-Rad Laboratories, Hercules, CA) with the following primer sets: RCP,
5'-GGATGTCTCCGAATCTTCCA-3' (forward) and 5'-CCGTCATCAGAGACAGCAAA-3' (reverse); MT1MMP, 5'-TTGGACTGTCAGGAATGAGG-3' (forward) and 5'-GCAGCACAAAATTCTCCGTG-3' (reverse); MMP2, 5'-ATGACAGCTGCACCACTGAG$3^{\prime}$ (forward) and 5'-AGTTCCCACCAACAGTGGAC-3' (reverse); MMP9, 5'-GTGCCATGTAAATCCCCACT$3^{\prime}$ (forward) and 5'-CTCCACTCCTCCCTTTCCTC-3' (reverse); uPA, 5'-GTGGCCAAAAGACTCTGAGG-3' (forward) and 5'-GCCGTACATGAAGCAGTGTG3' (reverse); Zeb1, 5'-AAGAATTCACAGTGGAGAGA AGCCA-3' (forward) and 5'-CGTTTCTTGCAGTTTG GGCATT-3' (reverse); and glyceraldehyde-3-phosphate dehydrogenase (GAPDH), 5'-A CAGTCAGCCGCATC TTCTT-3' (forward) and 5'-ACGACCAAATCCGTTGACTC-3' (reverse). The GAPDH gene was used as a control for calculating $\mathrm{dCt}$. The real-time PCR data were analyzed using the $2-(\mathrm{ddCt})$ method.

\section{Immunoblotting}

Immunoblotting was analyzed as previously described ${ }^{18}$. Briefly, the cell lysates were resolved by SDS-PAGE. PVDF membranes with proteins were blocked and incubated for $30 \mathrm{~min}$. Antibodies against RCP (1:1000, 12849S), Snail (1:1000, 3879S), N-cadherin (1:1000, 4061S), Zeb1 (1:1000, 3396S), p-EGFR (1:1000, 4407S), EGFR (1:1000, 2232 S), MT1-MMP (1:1000, 13130S) and nonphospho (NP) $\beta$-catenin $(1: 1000,19807 \mathrm{~S})$ were purchased from Cell Signaling Inc. (Danvers, MA). Antibodies against GAPDH (1:3000, sc-47724), Twist (1:1000, sc-15393), $\beta$-catenin (1:1000, sc-7963), and $\beta 1$ integrin (1:1000, sc53711) were purchased from Santa Cruz Biotechnology. An antibody against E-cadherin $(1: 1000,610182)$ was obtained from BD Biosciences (Franklin Lakes, NJ). The immunoreactive bands were exposed via ECL (Thermo Fisher Scientific Inc.) using an Amersham Imager 600 (GE Healthcare, Buckinghamshire, UK).

\section{Immunofluorescence}

Immunofluorescence was performed as previously described $^{19}$. Briefly, immunofluorescence detection was conducted with antibodies against RCP (1:500, Cell Signaling Technology), E-cadherin (1:500, Santa Cruz Biotechnology), $\beta 1$ integrin (1:500, Santa Cruz Biotechnology), MT1-MMP (1:500, Santa Cruz Biotechnology), and LAMP1 (1:500, Abcam, Cambridge, UK) overnight. The cells were washed with ice-cold PBS and incubated with Cy3conjugated goat anti-rabbit IgG (1:500, Jackson ImmunoResearch, PA), Cy2-conjugated goat anti-mouse IgG (1:500, Jackson ImmunoResearch) and Cy5-conjugated goat anti-rat IgG (1:500, Jackson ImmunoResearch). The nuclei of cells were marked with $4^{\prime}, 6^{\prime}$-diamidino-2-phenylindole (DAPI, Invitrogen). The cells were observed by confocal microscopy (x600, LSM710, Carl Zeiss). 


\section{In vitro invasion and wound healing assay}

Invasion and wound healing analyses were performed as previously described ${ }^{11,20}$. Briefly, $0.4 \times 10^{6}-1 \times 10^{6}$ cells were loaded in the upper well of the invasion chamber. After incubation for $13-15 \mathrm{~h}$ at $37^{\circ} \mathrm{C}$, the cells that invaded the membrane were fixed and stained with DiffQuik reagents (Sysmex Co., Kobe, Japan). The invaded cells were calculated by counting the number of cells in three arbitrary high-power fields for each replicate $(\times 200)$ with a light microscope. The results were derived from three independent experiments. For wound healing analysis, the cells were seeded in 6 -well plates for $24 \mathrm{~h}$ and then transfected with the indicated vector and siRNA. After serum starvation, the cells were scraped with a $200 \mu \mathrm{l}$ pipette tip. The gap distance of cells was observed after $24 \mathrm{~h}$ incubation in the same locations.

\section{Three-dimensional (3D) Matrigel culture}

Three-dimensional (3D) Matrigel culture was executed as previously described ${ }^{11}$. Briefly, cells were suspended in $2 \%$ Matrigel and put over a layer of polymerized $100 \%$ Matrigel at $1 \times 10^{4}$ cells $/ \mathrm{ml}$ in an eight-well chamber slide (Nunc, Littleton, CO). RPMI culture medium was replaced once every two days. Cultures were analyzed after 7 days of cultivation.

\section{Three-dimensional (3D) gel invasion assay}

3D gel invasion was analyzed as described previously with some modifications ${ }^{21-23}$. Cells were embedded in a mixture of $20 \%$ type I collagen (Nitta Gelatin Inc, Cellmatrix Type I-P, Japan) and Matrigel (BD Biosciences) in Transwell $(0.3 \mu \mathrm{m}$ pore size, Corning, Acton, MA). Oral CAFs and YD-10B cells were labeled with DiI (Oral CAFs; Thermo Fisher Scientific, Waltham, MA) and DiO (YD10B cells; Thermo Fisher Scientific), respectively. Oral CAFs $\left(2 \times 10^{4}\right)$ and YD-10B cells $\left(2 \times 10^{4}\right)$ were mixed in $200 \mu \mathrm{l}$ of medium (1:1 mixture of RPMI and medium of oral CAFs) supplemented with $0.2 \%$ FBS and plated on gels. The low chamber of the Transwell was filled with $800 \mu \mathrm{l}$ of the above medium with 10\% FBS. After 5 days, the embedded gel was sectioned without a fixture, and the cells were analyzed by fluorescence confocal microscopy. In these images, the distance of invaded cells was measured from eight different positions and calculated by the ZEN blue edition program of Carl Zeiss Microscopy $\mathrm{GmbH}$. The distance in $\mu \mathrm{m}$ was calculated as described previously $^{24}$.

\section{Cell viability}

The cell viability assay was determined as previously described $^{20,25}$. Briefly, cells were seeded in 96-well plates, serum-starved and treated with or without REV for $24 \mathrm{~h}$. The absorbance was calculated at $540 \mathrm{~nm}$ using a SYNERGY/ HTX ELISA plate reader (BioTek, Winooski, VT).

\section{Proteome extraction assay}

Proteome extraction assays were performed according to the manufacturer's protocol (Merck-539790). Briefly, YD-10B cells were transfected with the indicated vectors for $72 \mathrm{~h}$. After washing with cold washing buffer, the cells were added to fraction buffers to isolate supernatants with proteins in the cytosol (buffer I), membrane (buffer II) and nucleus (buffer III). Supernatants from buffers I and III were resolved by SDS-PAGE.

\section{Statistical analysis}

Data are shown as the means \pm standard deviation (SD). Differences between two groups were assessed with SigmaPlot software (Systat Software, San Jose, CA) using Student's $t$-test, and statistical significance was set at $p$-values less than 0.05 . Differences among three or more groups were estimated by analysis of variance followed by Bonferroni multiple comparison tests.

\section{Results \\ RCP induces OSCC EMT and invasiveness}

To determine the role of RCP in OSCC invasiveness, we first determined the level of RCP expression in OSCC. We observed that all tested OSCCs express detectable amounts of RCP (Supplementary Fig. 1). In addition, ectopic expression of RCP significantly increased their invasiveness (Fig. 1a). By contrast, silencing of RCP expression markedly attenuated the invasiveness of OSCC cells (Fig. 1b). Moreover, we observed the increased growth of YD-10B cells on 3D Matrigel when they were transfected with RCP (Fig. 1c). To examine the effects of $\mathrm{RCP}$ on coordinated invasion by oral CAFs and cancer cells, a 3D gel invasion assay was utilized, which reflects cancer invasion in vivo. Cells were labeled with distinguishable fluorescent dyes and placed on top of a gel containing type I collagen and Matrigel (Fig. 1d). Interestingly, compared to the vector control, overexpression of RCP resulted in a higher invasive potential in cells cocultured with oral cancer-associated fibroblasts (CAFs) in 3D Matrigel (Fig. 1e), indicating that RCP reorganizes the microenvironment to augment the invasiveness of OSCC. Furthermore, immunofluorescence analysis showed that RCP induces EMT in OSCC. RCP efficiently reduced the E-cadherin expression of YD-10B cells, while transfection of the cells with RCP siRNA dramatically increased Ecadherin expression in YD-38 cells (Fig. 1f, g). Therefore, these data imply the critical role of RCP in OSCC EMT and invasion.

\section{Zeb1 is important for RCP-induced OSCC invasion}

We next determined whether modulation of the expression level of RCP alters the expression of EMT-TFs in OSCC. Ectopic expression of RCP in YD-10B cells prominently increased N-cadherin and Zeb1 expression 


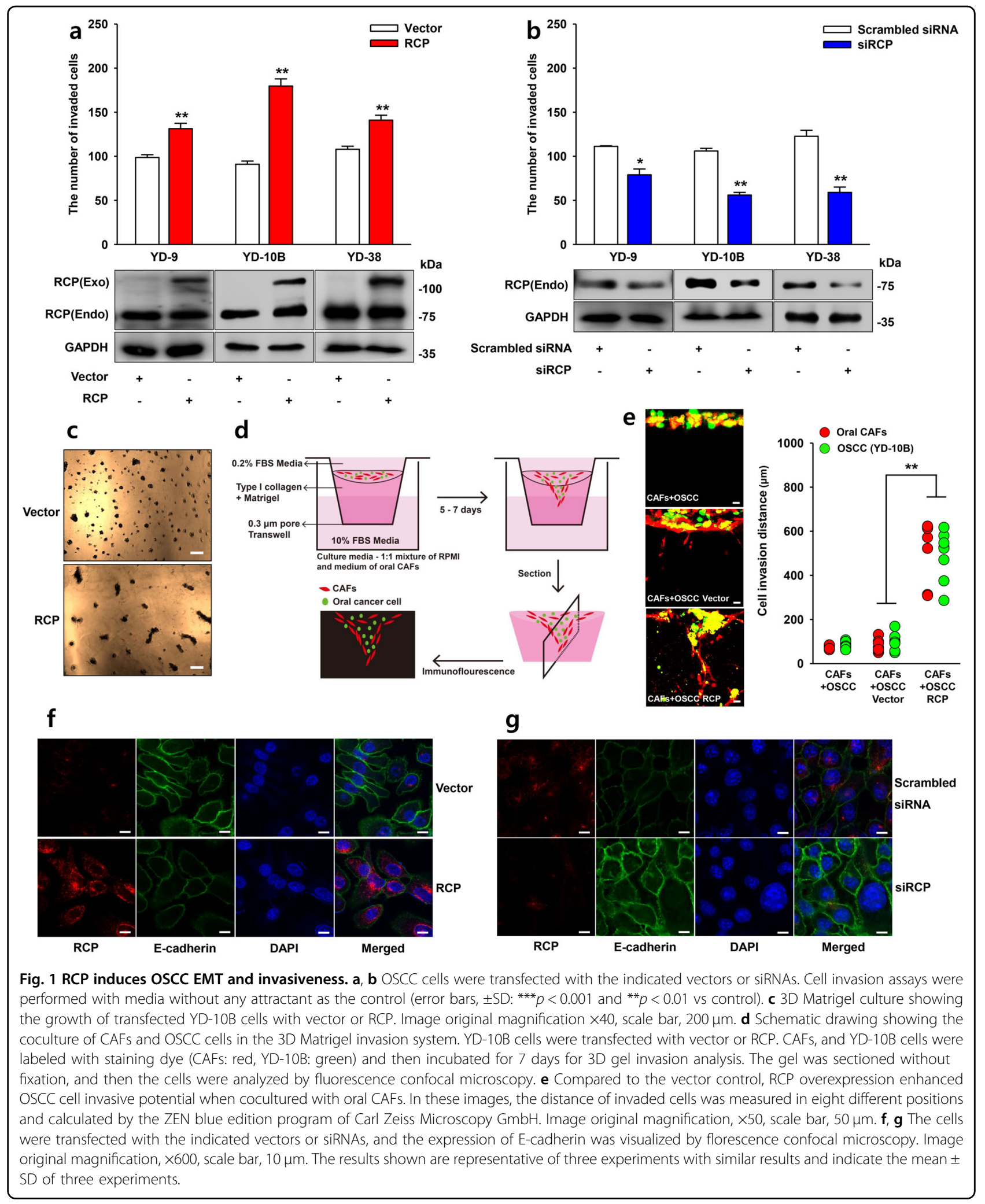

(Fig. 2a). In contrast, silencing of RCP expression in YD38 cells reduced and induced the expression of these EMT-TFs and E-cadherin, respectively (Fig. 2b). To determine the roles of these EMT-TFs in RCP-induced OSCC invasion, we transfected YD-10B cells with siRNAs targeting $\mathrm{N}$-cadherin and Zeb1 and observed that 


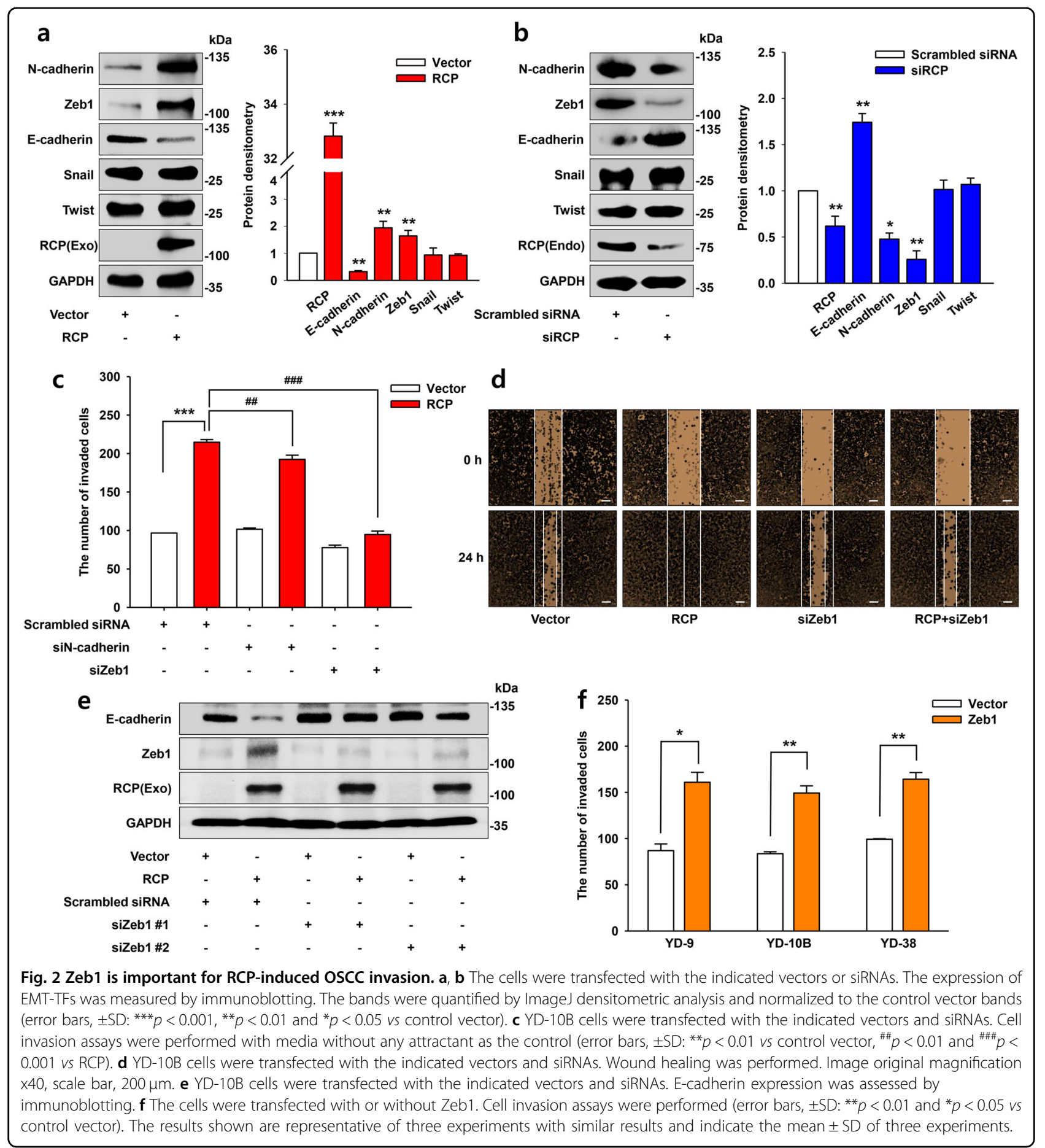

silencing Zeb1 expression shows more dramatic inhibition of RCP-induced YD-10B cell invasion than silencing $\mathrm{N}$-cadherin (Fig. 2c). Likewise, Zeb1 siRNA markedly inhibited RCP-induced cancer cell migration (Fig. 2d). In addition, siRNAs targeting Zeb1 attenuated the reduction in E-cadherin expression induced by RCP (Fig. 2e). Furthermore, ectopic expression of Zeb1-induced OSCC invasion (Fig. 2f), indicating that Zeb1 is sufficient to increase OSCC invasion. Therefore, these data provide evidence that Zeb1 is associated with RCP-induced OSCC EMT and invasion.

\section{RCP induces Zeb1 expression through the $\beta 1$ integrin/ EGFR signaling axis}

Since RCP has been known to exert its oncogenic property by recycling $\beta 1$ integrin and thereby EGFR 


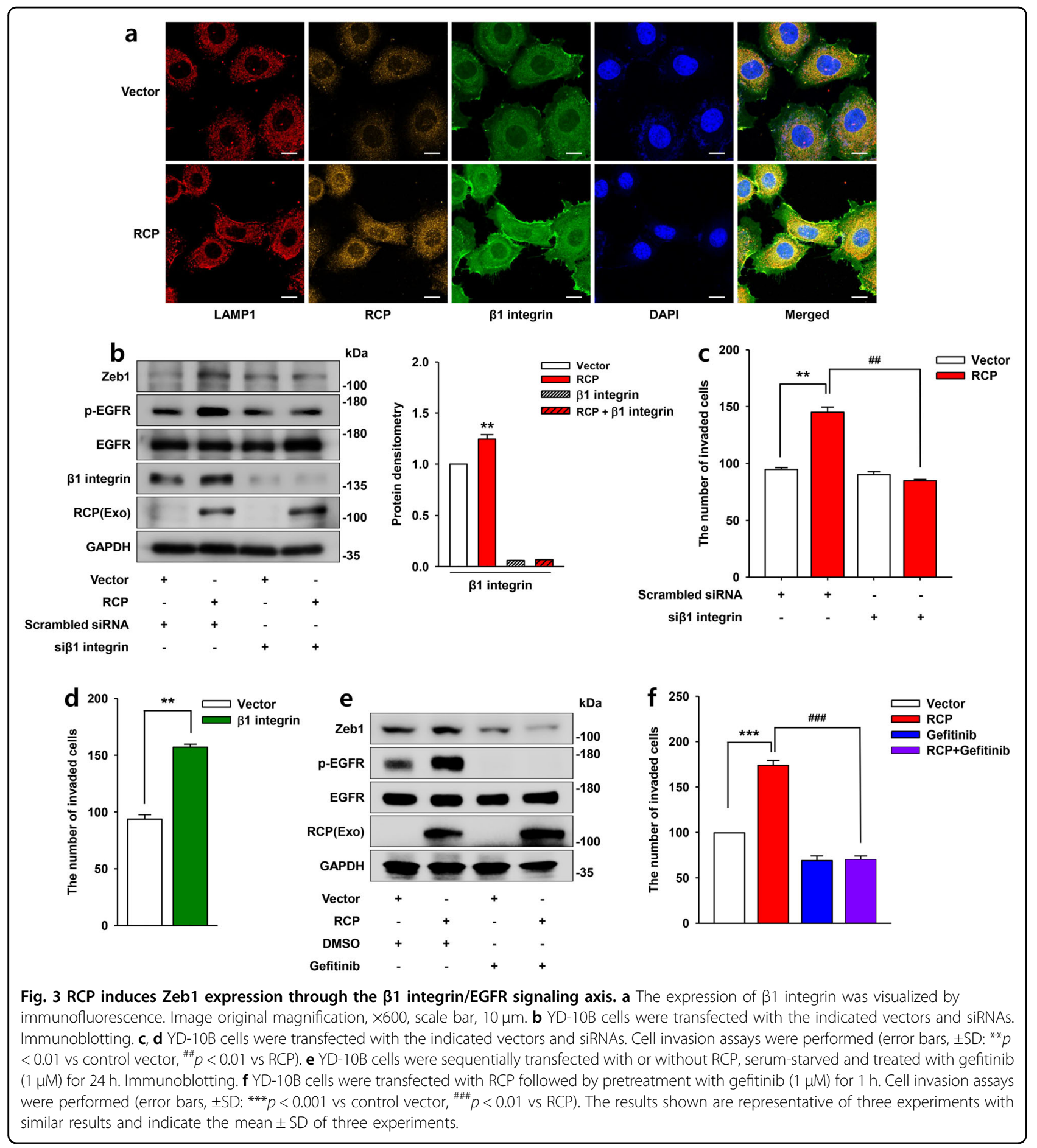

activation $^{6}$, we compared the level of expression and the location of $\beta 1$ integrin in the cells overexpressing RCP and the control. Immunofluorescence data showed that RCP markedly upregulates the level of $\beta 1$ integrin expression in the plasma membrane of OSCC compared to the control. RCP was mainly detected in the cytosol. Congruently, we observed increased microspikes stained with $\beta 1$ integrin at the plasma membrane by RCP (Fig. 3a). Furthermore, immunoblotting analysis revealed increased levels of $\beta 1$ integrin expression and phospho-EGFR by RCP. However, silencing $\beta 1$ integrin expression dramatically decreased RCP-induced Zeb1 expression (Fig. 3b) as well as OSCC invasion (Fig. 3c). In addition, overexpression of $\beta 1$ integrin-induced OSCC invasion 
(Fig. 3d). Moreover, treatment of the cells with a specific pharmacological inhibitor of EGFR, gefitinib, abolished RCP-induced Zeb1 expression (Fig. 3e) and OSCC invasion (Fig. 3f). Therefore, these data suggest that Zeb1 is critical for RCP-induced OSCC invasion through $\beta 1$ integrin and EGFR activation.

\section{The $\beta$-catenin/TCF4 complex mediates RCP-induced Zeb1 expression}

Given that $\beta$-catenin with TCF4 binds to the Zeb1 promoter and induces its expression as well as tumor $\operatorname{progression}^{26}$, we next explored whether $\beta$-catenin is implicated in Zeb1 expression in OSCC. RCP markedly increased active $\beta$-catenin expression (Fig. 4a). However, silencing of $\beta$-catenin expression or incubation of the cells with a selective pharmacological inhibitor of $\beta$-catenin, FH535, profoundly reduced RCP-induced Zeb1 expression (Fig. 4a, c) and invasion (Fig. 4b, d). In addition, we noticed that RCP increases the level of active $\beta$-catenin expression in the nucleus (Fig. 4e). Interestingly, overexpression of $\beta 1$ integrin also upregulated active $\beta$-catenin expression (Fig. 4f), confirming that RCP, and thereby $\beta 1$

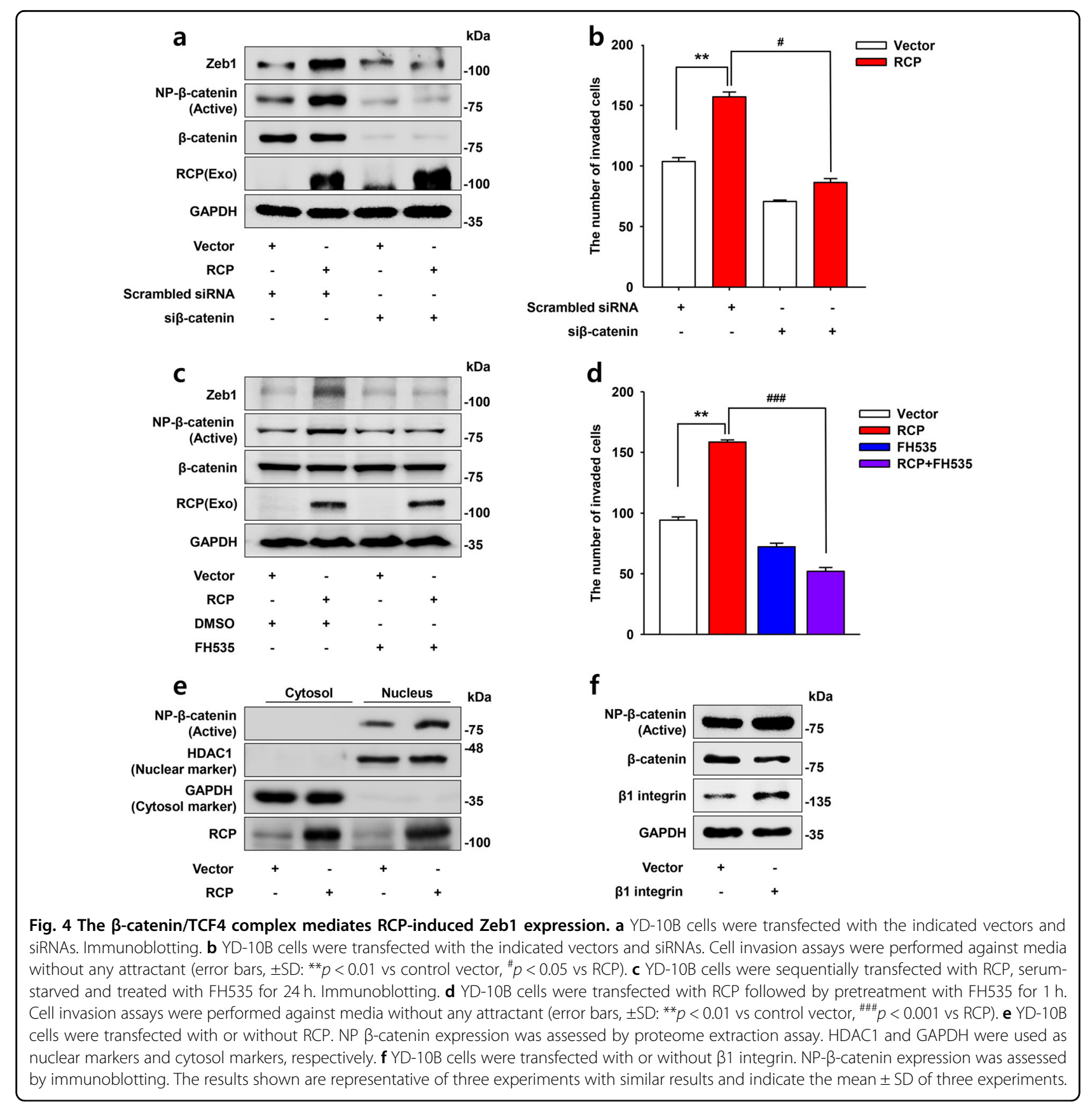




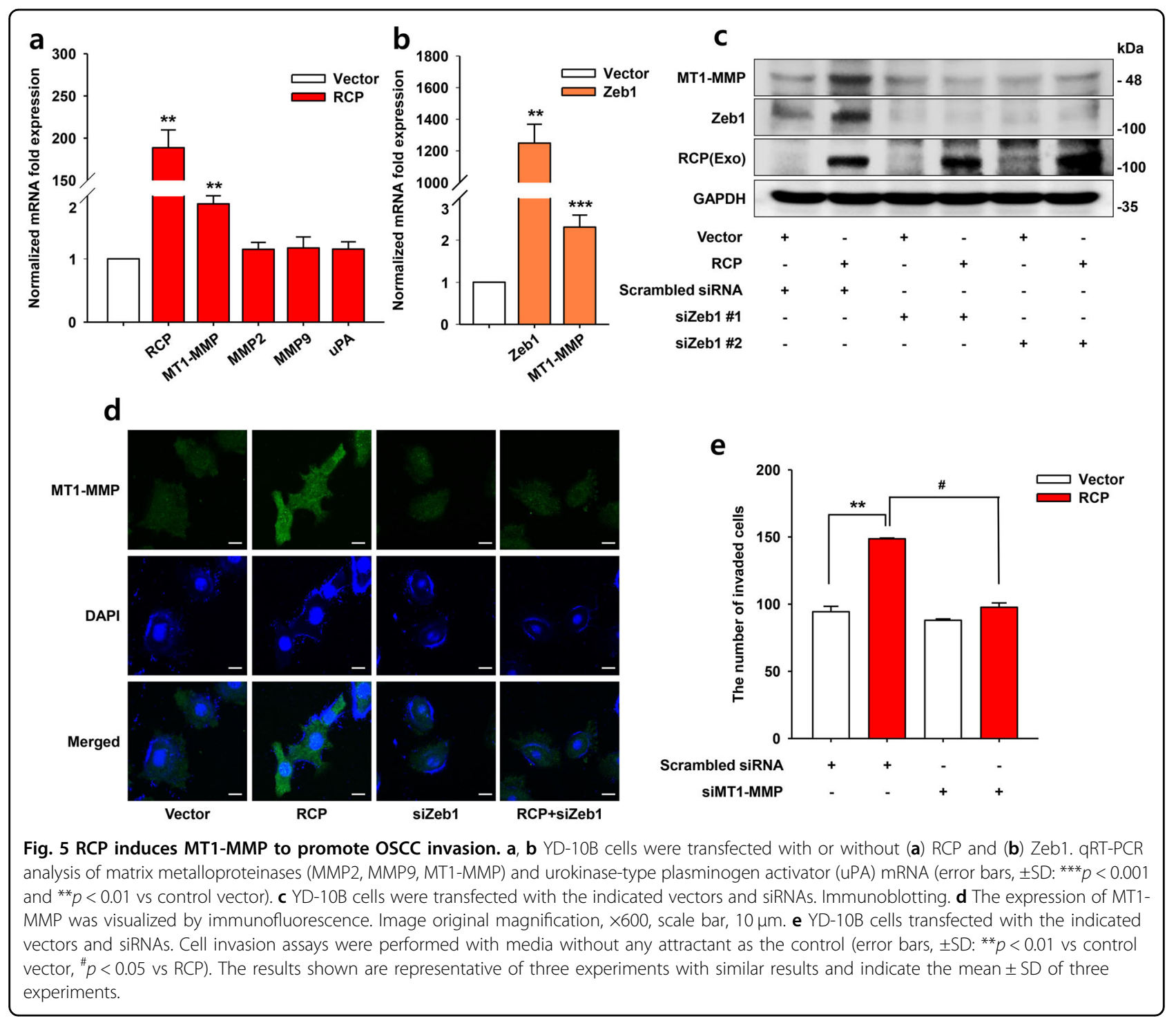

integrin, induce translocation of $\beta$-catenin to upregulate Zeb1 expression. Therefore, these data indicate that $\beta$-catenin located downstream of $\beta 1$ integrin is implicated in RCP-induced Zeb1 expression.

\section{RCP induces MT1-MMP for OSCC invasion}

To specify a critical factor for RCP-induced and thereby Zeb1-induced OSCC invasion, we determined whether RCP modulates the transcript expression of several important proteolytic enzymes for OSCC invasiveness. RCP increased only the MT1-MMP transcript among the tested factors (Fig. 5a). Consistent with these results, the ectopic expression of Zeb1-induced MT1-MMP transcript expression (Fig. 5b). In addition, silencing Zeb1 expression significantly inhibited RCP-induced MT1MMP expression (Fig. 5c, d), suggesting that Zeb1 mediates MT1-MMP expression in OSCC. Notably, silencing of MT1-MMP expression ablated RCP-induced OSCC invasion (Fig. 5e). Therefore, these results indicate that MT1-MMP is located downstream of Zeb1 and is necessary for RCP-induced OSCC invasion.

\section{REV mitigates RCP-induced OSCC invasion}

Since REV has strong anti-metastatic effects on various cancer cells $^{27}$, we assessed whether REV attenuates RCPinduced OSCC invasion. YD-10B cells had survival rates of $85 \%$ or more with REV concentrations up to $25 \mu \mathrm{M}$ (Supplementary Fig. 2), indicating that REV at these concentrations does not cause any significant growth inhibition of YD-10B cells. Moreover, REV attenuated OSCC cell invasion in a dose-dependent manner (Fig. 6a). Treatment of these cells with REV dramatically abolished RCP-induced Zeb1 expression and MT1-MMP expression (Fig. 6b) as well as OSCC invasiveness (Fig. 6c). REV 


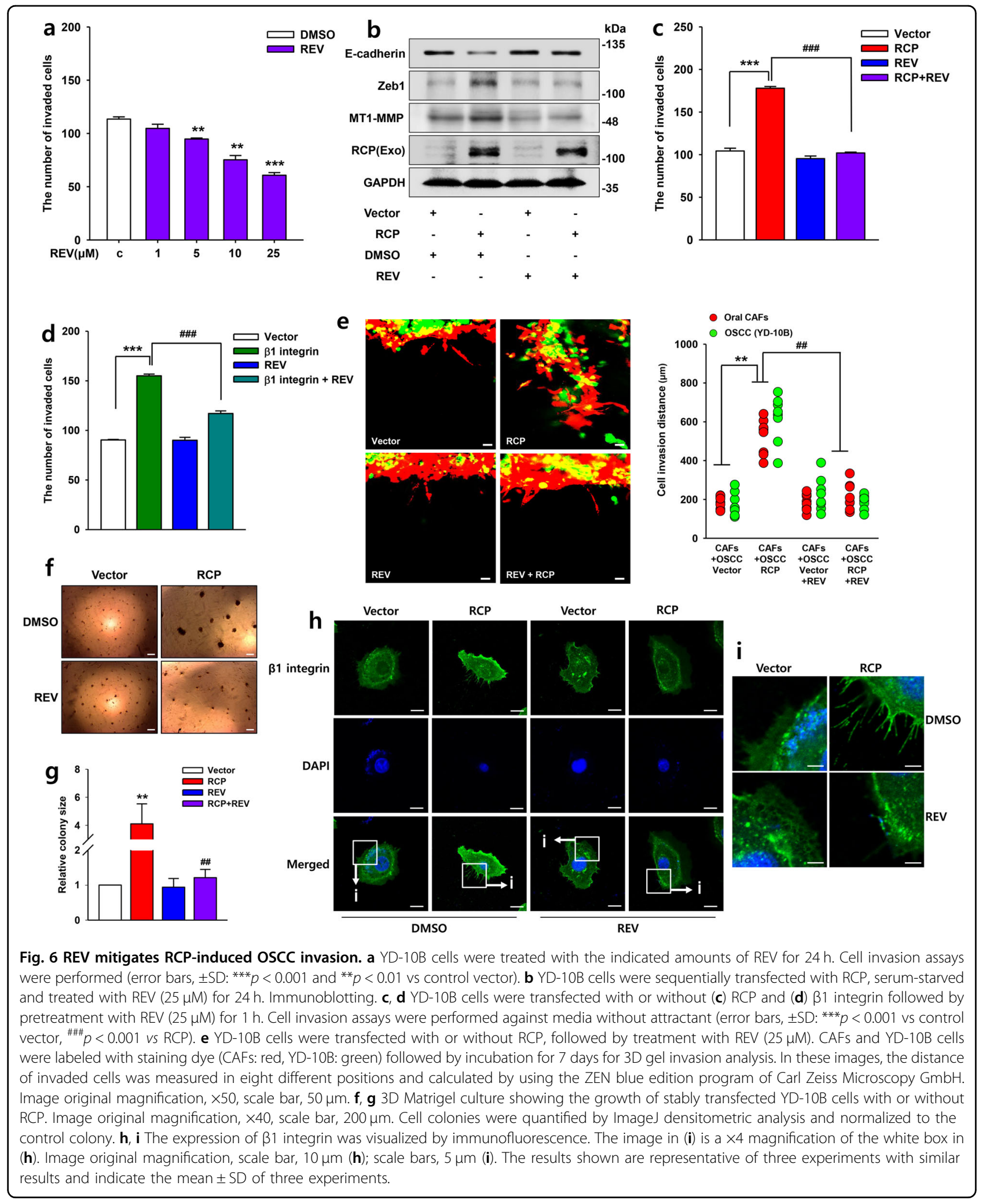

also efficiently attenuated $\beta 1$ integrin-induced OSCC invasion (Fig. 6d). Consistent with these findings, REV ablated RCP-induced OSCC invasion compared to vector transfectant when the cells were co-cultured with oral CAFs (Fig. 6e), implying that REV suppresses RCPinduced changes in the tumor microenvironment of 
OSCC. Furthermore, REV efficiently blocked the RCPinduced growth of OSCC in 3D Matrigel (Fig. 6f, g). Moreover, we noticed that REV intensely blocks RCPinduced $\beta 1$ integrin expression and filopodia formation at the leading edge of OSCC (Fig. 6h, i). Therefore, these data indicate that REV inhibits RCP-induced salvage of $\beta 1$ integrin from lysosomal degradation and thus reduces Zeb1 expression, resulting in attenuation of OSCC invasiveness.

\section{Discussion}

Accumulating evidence has suggested the critical role of RCP in endosome recycling and cancer progression. While the majority of studies have pointed out the oncogenic function of RCP, a recent report showed that RCP exerts a tumor suppressive role in mammary tumors through destabilizing $\mathrm{ErBB}{ }^{15}$, indicating the types of cancer- and context-dependent characteristics of RCP in cancer progression. In the present study, we show for the first time that RCP positively regulates OSCC invasiveness through Zeb1 and MT1-MMP expression. Furthermore, we provide evidence that REV efficiently attenuates RCPinduced OSCC invasion through the downregulation of $\beta 1$ integrin and consequent Zeb1 expression.

RCP plays a prominent role in the invasion and metastasis of various cancers through recycling endosomes and thereby recapitulating integrins and growth factor receptors ${ }^{8}$. Indeed, we recently provide evidence that RCP stabilizes $\beta 1$ integrin from lysosomal degradation and activation of the EGFR downstream signaling cascade, culminating in aggravation of cancer invasion and metastasis ${ }^{11}$. In the present study, we extended our study to investigate the role of RCP in the invasiveness of one of the aggressive types of HNSCC, OSCC, and uncovered that RCP induces OSCC invasion (Fig. 1a, b). In addition, RCP caused a dramatic increase in filopodia filled with $\beta 1$ integrin around the plasma membrane (Figs. $3 a$ and $6 i)$, indicating the role of RCP in OSCC invasiveness. Furthermore, we observed enhanced invasiveness in RCP-overexpressing OSCC cells compared to vectortransfected cells when they were cocultured with oral CAFs (Figs. 1e and 6e), suggesting that RCP enables OSCC to communicate with oral CAFs in the tumor microenvironment and promotes OSCC invasion. It might be possible that RCP causes OSCC cells to secrete factors activating nearby oral CAFs to promote OSCC invasion. The underlying mechanism by which RCP connects OSCC cells with oral CAFs to regulate OSCC invasion is under current investigation.

Zeb1 is a well-known EMT-TF associated with tumor progression. In addition to participating in the invasion and metastatic dissemination of cancer cells, Zeb1 is implicated in malignant tumor formation, cancer stem- ness, and resistance to treatment ${ }^{28}$. Unlike in other types of cancers, the role of Zeb1 in HNSCC and OSCC is now receiving attention. Recently, Zeb1 was identified as an indicator for recurrent $\mathrm{OSCC}^{29}$, and blocking Zeb1 resulted in regression of OSCC metastasis ${ }^{30}$. In addition, a higher level of Zeb1 expression was detected in advanced tongue squamous cell carcinoma compared with neighboring normal epithelia ${ }^{31}$. Furthermore, the association of Zeb1 expression with OSCC invasion was identified through RNA-binding protein quaking $(\mathrm{QKI})^{32}$. In the present study, we underscore the important role of Zeb1 in RCP-induced OSCC invasion. Indeed, Zeb1 was sufficient to increase OSCC invasion (Fig. 2f). Conversely, silencing Zeb1 expression attenuated RCP-induced OSCC invasion (Fig. 2c), unveiling the critical role of Zeb1 in OSCC progression and an important target for OSCC patients. In addition, we identified that Zeb1 is located downstream of the $\beta 1$ integrin/EGFR signaling axis (Fig. $3 \mathrm{~b}, \mathrm{e})$. Among several proteolytic enzymes implicated in cancer invasion, RCP and Zeb1 increase MT1-MMP, which is critical for RCP-induced OSCC invasion. In support of our results, MT1-MMP is an important proteolytic enzyme for OSCC EMT and invasion ${ }^{33,34}$.

The naturally occurring polyphenolic compound REV (trans-3,4,5-trihydroxystilbene) has multiple anti-metastatic effects in various cancer cells ${ }^{27}$. REV has been shown to suppress OSCC $\mathrm{EMT}^{35}$ and invasion ${ }^{36}$. In this study, we elucidated an additional mechanism by which REV attenuates OSCC progression. We provide evidence that REV blocks the recycling of $\beta 1$ integrin to the plasma membrane and consequently inactivates EGFR and the downstream signaling cascade for Zeb1 expression (Fig. 6b) and OSCC invasion (Fig. 6c, d). In support of our data, REV has been shown to inhibit Zeb1 expression to reduce the myofibroblast activity of buccal mucosal fibroblasts ${ }^{37}$. In addition, our data show for the first time that REV suppresses RCP-induced OSCC filopodia formation (Fig. 6h, i), which is in good accordance with a previous report showing REV-induced inhibition of filopodia formation in renal cell carcinoma cells ${ }^{38}$. In view of our current results, we present a working model (Fig. 7) showing that RCP promotes OSCC invasion by promoting the recycling of $\beta 1$ integrin and the activation of the EGFR/ $\beta$-catenin/Zeb1/MT1-MMP signaling axis and also shows that REV inhibits RCPinduced OSCC invasion.

\footnotetext{
Acknowledgements

We would like to thank Dr. Jim Norman (Beaston Cancer Institute, Glasgow, UK) who provided RCP and Dr. Y.S. Lee (Ewha Woman's University, Seoul, Korea) who provided $\beta 1$ integrin. We also want to thank Dr. X.L. Zhang and Dr. J.I. Yook at Yonsei University College of Dentistry for oral CAFs and YD-10B cells, respectively. This work is supported by a grant from the Basic Science Research Program through the National Research Foundation of Korea (NRF) funded by the Ministry of Education, Science and Technology (NRF-2017R1E1A1A01074091).
} 


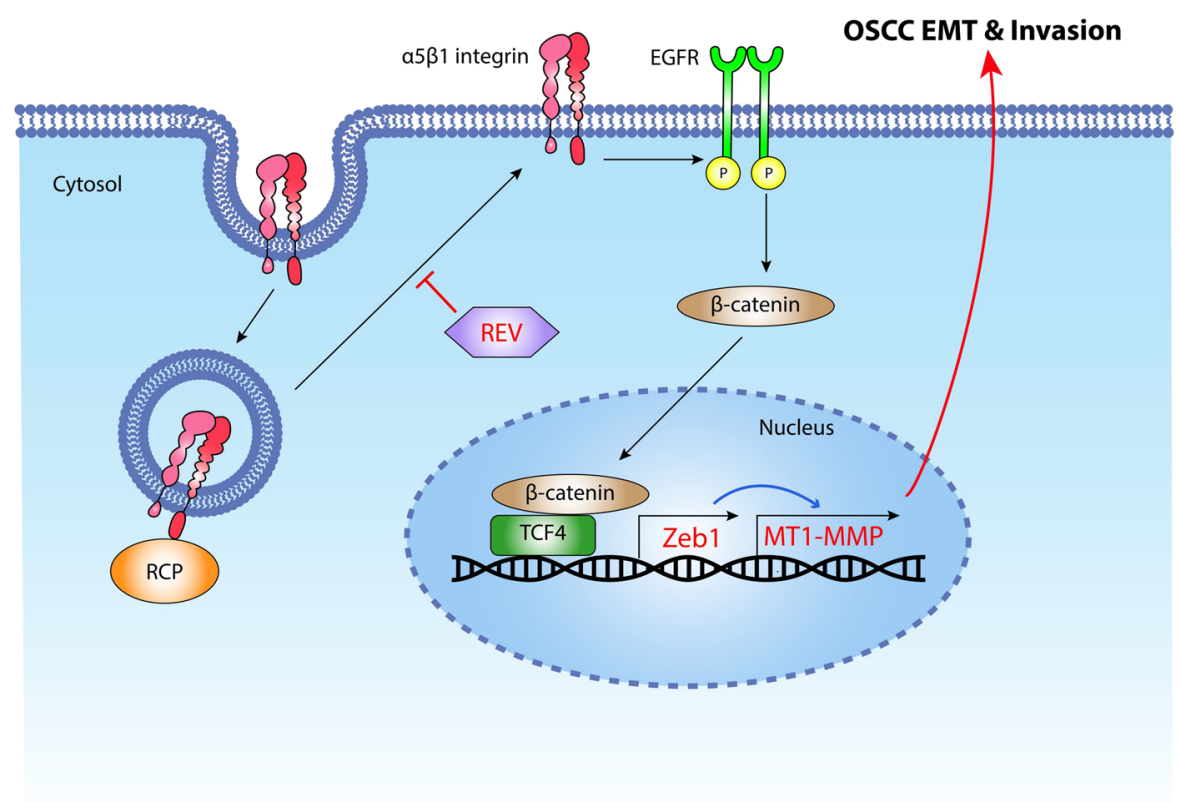

Fig. 7 Working model showing how RCP promotes OSCC invasion, which is reversed by REV. RCP induces Zeb1 and MT1-MMP expression through the $\beta 1$ integrin/EGFR/ $\beta$-catenin signaling axis to promote OSCC invasion. REV efficiently blocks RCP-induced $\beta 1$ integrin relocation to the plasma membrane and EGFR activation, resulting in suppression of RCP-induced OSCC invasion.

\section{Author contributions}

Conception and design of the study: Hoi Young Lee, and Chang Gyo Park. Data curation: Jin Young Kim, Kyung Hwa Cho, and Bo Young Jeong. Formal analysis: Jing Young Kim. Investigation: Jin Young Kim, and Bo Young Jeong. Methodology: Jin Young Kim, Kyung Hwa Cho, and Bo Young Jeong.

Supervision: Hoi Young Lee and Chang Gyo Park. Writing of original draft: Hoi Young Lee and Jin Young Kim. Manuscript editing: Hoi Young Lee, Chang Gyo Park, and Jin Young Kim All authors discussed the results and commented on the paper.

\section{Conflict of interest}

The authors declare that they have no conflict of interest.

\section{Publisher's note}

Springer Nature remains neutral with regard to jurisdictional claims in published maps and institutional affiliations.

Supplementary information accompanies this paper at https://doi.org/ 10.1038/s12276-020-0474-1.

Received: 4 December 2019 Revised: 29 April 2020 Accepted: 15 June 2020. Published online: 30 July 2020

\section{References}

1. Zhang, J. et al. Overexpression of Rab25 contributes to metastasis of bladder cancer through induction of epithelial-mesenchymal transition and activation of Akt/GSK-3beta/Snail signaling. Carcinogenesis $\mathbf{3 4}$ 2401-2408 (2013).

2. Rakha, E. A. et al. Biologic and clinical characteristics of breast cancer with single hormone receptor positive phenotype. J. Clin. Oncol. 25, 4772-4778 (2007).

3. Bray, F. et al. Global cancer statistics 2018: GLOBOCAN estimates of incidence and mortality worldwide for 36 cancers in 185 countries. CA Cancer J. Clin. 68 , 394-424 (2018).
4. Park, H. S. et al. High EGFR gene copy number predicts poor outcome in triple-negative breast cancer. Mod. Pathol. 27, 1212-1222 (2014).

5. Kassam, F. et al. Survival outcomes for patients with metastatic triple-negative breast cancer: implications for clinical practice and trial design. Clin. Breast Cancer 9, 29-33 (2009).

6. Caswell, P. T. et al. Rab-coupling protein coordinates recycling of alpha5beta1 integrin and EGFR1 to promote cell migration in 3D microenvironments. J. Cell Biol. 183, 143-155 (2008).

7. Lindsay, A. J. et al. Rab coupling protein (RCP), a novel Rab4 and Rab11 effector protein. J. Biol. Chem. 277, 12190-12199 (2002).

8. Cho, K. H. \& Lee, H. Y. Rab25 and RCP in cancer progression. Arch. Pharmacal Res. 42, 101-112 (2019).

9. Dai, Y. et al. Increased expression of Rab coupling protein in squamous cell carcinoma of the head and neck and its clinical significance. Oncol. Lett. 3, 1231-1236 (2012).

10. Paul, N. R. et al. alpha5beta1 integrin recycling promotes Arp2/3-independent cancer cell invasion via the formin FHOD3. J. Cell Biol. 210, 1013-1031 (2015).

11. Hwang, M. H. et al. RCP induces Slug expression and cancer cell invasion by stabilizing beta1 integrin. Oncogene 36, 1102-1111 (2017).

12. Gundry, C. et al. Phosphorylation of Rab-coupling protein by LMTK3 controls Rab14-dependent EphA2 trafficking to promote cell:cell repulsion. Nat. Commun. 8, 14646 (2017).

13. Novo, D. et al. Mutant p53s generate pro-invasive niches by influencing exosome podocalyxin levels. Nat. Commun. 9, 5069 (2018).

14. Rainero, E. et al. Diacylglycerol kinase alpha controls RCP-dependent integrin trafficking to promote invasive migration. J. Cell Biol. 196, 277-295 (2012).

15. Boulay, P. L. et al. Rab11-FIP1C is a critical negative regulator in ErbB2 mediated mammary tumor progression. Cancer Res. 76, 2662-2674 (2016).

16. Miller, K. D et al. A multicenter phase II trial of ZD6474, a vascular endothelia growth factor receptor-2 and epidermal growth factor receptor tyrosine kinase inhibitor, in patients with previously treated metastatic breast cancer. Clin. Cancer Res. 11, 3369-3376 (2005).

17. Jeong, B. Y. et al. Rab25 augments cancer cell invasiveness through a beta1 integrin/EGFRVEGF-A/Snail signaling axis and expression of fascin. Exp. Mol. Med. 50, e435 (2018).

18. Cho, K. H. et al. The YB-1/EZH2/amphiregulin signaling axis mediates LPAinduced breast cancer cell invasion. Arch. Pharmacal Res. 42, 519-530 (2019). 
19. Choe, S. R. et al. RCP induces FAK phosphorylation and ovarian cancer cell invasion with inhibition by curcumin. Exp. Mol. Med. 50, 52 (2018)

20. Seo, J. H. et al. Lysophosphatidic acid induces STAT3 phosphorylation and ovarian cancer cell motility: their inhibition by curcumin. Cancer Lett. $\mathbf{2 8 8}$ 50-56 (2010).

21. Kim, Y. H. et al. Senescent tumor cells lead the collective invasion in thyroid cancer. Nat. Commun. 8, 15208 (2017).

22. Satoyoshi, R. et al. Asporin activates coordinated invasion of scirrhous gastric cancer and cancer-associated fibroblasts. Oncogene 34, 650-660 (2015).

23. Tanaka, $M$. et al. Identification of anti-cancer chemical compounds using Xenopus embryos. Cancer Sci. 107, 803-811 (2016).

24. Vehlow, A. et al. Adhesion- and stress-related adaptation of glioma radiochemoresistance is circumvented by beta1 integrin/JNK co-targeting. Oncotarget 8, 49224-49237 (2017).

25. Kim, Y. N. et al. Resveratrol suppresses breast cancer cell invasion by inactivating a RhoAYAP signaling axis. Exp. Mol. Med. 49, e296 (2017).

26. Sanchez-Tillo, E. et al. beta-catenin/TCF4 complex induces the epithelial-tomesenchymal transition (EMT)-activator ZEB1 to regulate tumor invasiveness. Proc. Natl Acad. Sci. USA 108, 19204-19209 (2011).

27. Kundu, J. K. \& Surh, Y. J. Cancer chemopreventive and therapeutic potential of resveratrol: mechanistic perspectives. Cancer Lett. 269, 243-261 (2008).

28. Caramel, J. et al. Pleiotropic Roles for ZEB1 in Cancer. Cancer Res. 78, 30-35 (2018).

29. Ho, C. M. et al. ZEB1 as an indicator of tumor recurrence for areca quid chewing-associated oral squamous cell carcinomas. J. Oral. Pathol. Med. 44, 693-698 (2015)
30. Wu, B. et al. MiRNA-101 inhibits oral squamous-cell carcinoma growth and metastasis by targeting zinc finger E-box binding homeobox 1. Am. J. Cancer Res. 6, 1396-1407 (2016).

31. Kurihara, K. et al. Expression of BMI1 and ZEB1 in epithelial-mesenchymal transition of tongue squamous cell carcinoma. Oncol. Rep. 34, 771-778 (2015).

32. Kim, E. J. et al. QKl, a miR-200 target gene, suppresses epithelial-tomesenchymal transition and tumor growth. Int J. Cancer 145, 1585-1595 (2019).

33. Yang, C. C. et al. Membrane type 1 matrix metalloproteinase induces an epithelial to mesenchymal transition and cancer stem cell-like properties in SCC9 cells. BMC cancer 13, 171 (2013).

34. Li, Y. Y. et al. Podoplanin promotes the invasion of oral squamous cell carcinoma in coordination with MT1-MMP and Rho GTPases. Am. J. Cancer Res. 5, 514-529 (2015).

35. Kim, S. E. et al. Resveratrol induces mitochondrial apoptosis and inhibits epithelial-mesenchymal transition in oral squamous cell carcinoma cells. Nutr. Cancer 70, 125-135 (2018).

36. Lin, F. Y. et al. Resveratrol suppresses TPA-induced matrix metalloproteinase-9 expression through the inhibition of MAPK pathways in oral cancer cells. Oral. Pathol. Med. 44, 699-706 (2015).

37. Chang, Y. C. et al. Resveratrol suppresses myofibroblast activity of human buccal mucosal fibroblasts through the epigenetic inhibition of ZEB1 expression. Oncotarget 7, 12137-12149 (2016).

38. Zhao, Y. et al. Resveratrol inhibits proliferation, migration and invasion via Akt and ERK1/2 signaling pathways in renal cell carcinoma cells. Biomed. Pharmacother. 98, 36-44 (2018). 